CERN-TH/98-357

FNT/T-98/12

\title{
Six-fermion production and Higgs boson physics at future $e^{+} e^{-}$colliders
}

\author{
Fabrizio GANGEMI ${ }^{1,2}$, Guido MONTAGNA ${ }^{1,2}$, Mauro MORETTI ${ }^{3,4}$, \\ Oreste NICROSINI ${ }^{2,1}$ and Fulvio PICCININI ${ }^{2,1}$
}

${ }^{1}$ Dipartimento di Fisica Nucleare e Teorica, Università di Pavia, Italy

${ }^{2}$ Istituto Nazionale di Fisica Nucleare, Sezione di Pavia, Italy

${ }^{3}$ CERN - Theory Division, Geneva, Switzerland

${ }^{4}$ Dipartimento di Fisica, Università di Ferrara and INFN, Sezione di Ferrara, Italy

\begin{abstract}
The six-fermion production processes $e^{+} e^{-} \rightarrow q \bar{q} l^{+} l^{-} \nu \bar{\nu}$, with all the lepton flavours and $q=u, d, c, s$, relevant to the study of the intermediate-mass Higgs boson at future $e^{+} e^{-}$linear colliders, are analysed. A Monte Carlo program, taking into account the whole set of tree-level scattering amplitudes and the relevant radiative effects, is developed to provide integrated cross sections and generation of unweighted events. The complete calculation is compared with the available results of real Higgs production, and the opportunities of precision studies with event generation are discussed, demonstrating the relevance of a full six-fermion calculation. Numerical results for integrated cross sections with various kinematical cuts and including radiative effects are given and commented. In the analysis of event samples, several distributions are studied and found to be sensitive to the presence and to the properties of the Higgs boson.
\end{abstract}

PACS: 02.70.Lq, 13.85.Hd, 14.80.Bn

Keywords: electron-positron collisions, six fermions, Higgs boson, Monte Carlo. 


\section{Introduction}

The search for the Higgs boson and the study of its properties will be among the most important tasks of elementary particle physics at future $e^{+} e^{-}$linear colliders at the TeV scale (NLC) [1].

The research carried on at present colliders can explore an interval of Higgs masses below $\sim 100 \mathrm{GeV}$ at LEP2 [2] or possibly $120-130 \mathrm{GeV}$ at the upgraded Tevatron [3]. The remaining mass range, up to $\sim 800 \mathrm{GeV}$, will be in the reach of the future colliders LHC [4] and NLC. In particular the precision studies that will be possible in the clean environment of NLC will be of great help in the determination of the Higgs boson properties.

A range of particular interest for the Higgs mass is between 100 and 200 $\mathrm{GeV}$, as many arguments both of experimental and of theoretical nature indicate. Indeed a lower limit of $\sim 90 \mathrm{GeV}$ is given by recent results in the direct search at LEP2 [2], while from fits to electroweak precision data an upper limit of $\sim 280 \mathrm{GeV}$ at $95 \%$ C.L. is obtained [5].

In this range two mass intervals may be considered: for $m_{H} \leq 140 \mathrm{GeV}$ the Higgs decays mainly into $b \bar{b}$ pairs, while for $m_{H} \geq 140 \mathrm{GeV}$ the decays into $W W$ and $Z Z$ pairs become dominant. Therefore in the first case the mechanisms of Higgs production relevant to $e^{+} e^{-}$colliders, Higgs-strahlung and $V V(V=W, Z)$ fusion, give rise to signatures that contain four fermions in the final state, which have been extensively studied in the recent past [6][9]. In the second case, in which $m_{H} \geq 140 \mathrm{GeV}$, six fermions are produced in the final state. More generally, six-fermion $(6 f)$ final states come from other relevant processes at NLC, such as $t \bar{t}$ and three-gauge-boson production [10].

As shown by complete four-fermion calculations for $W W$ and light Higgs physics at LEP2 [6]-[9, 11, 12], full calculations of $6 f$ production processes allow one to keep phenomenologically relevant issues under control, such as off-shellness and interference effects, background contributions and spin correlations. Some calculations of such processes $e^{+} e^{-} \rightarrow 6 \mathrm{f}$ have recently been performed [13]-[16], with regard to top-quark, Higgs boson and $W W Z$ physics at NLC. Moreover recent progress in the calculation of processes $e^{+} e^{-} \rightarrow 6$ jets [17] and of $2 \rightarrow$ up to 8 partons QCD amplitudes [18, 19] should be mentioned for their relevance in QCD tests at lepton and hadron machines. These calculations rely upon different computational techniques, such as helicity amplitude methods for the evaluation of the very large number of Feynman diagrams associated to the process under examination, or 
iterative numerical algorithms, where the transition amplitudes are calculated numerically without using Feynman diagrams.

Concerning Higgs physics, an analysis of the processes $e^{+} e^{-} \rightarrow \mu^{+} \mu^{-} u \bar{d} \tau^{-} \bar{\nu}_{\tau}$ and $e^{+} e^{-} \rightarrow \mu^{+} \mu^{-} u \bar{d} e^{-} \bar{\nu}_{e}$ has been performed in ref. [15], where the Higgs can be produced by Higgs-strahlung and the subsequent decay proceeds through $W^{+} W^{-}$pairs. Special attention has been devoted to the calculation of the Higgs boson signal and of its Standard Model background, with special emphasis on the determination and analysis of angular correlation variables, particularly sensitive to the presence and to the spinless nature of the Higgs particle. The $6 f$ final states, where the Higgs signal gives rise to two charged currents, have also been considered in ref. [13], studying cross sections and invariant mass distributions for the processes $e^{+} e^{-} \rightarrow f \bar{f} q \bar{q}^{\prime} f^{\prime} \bar{f}^{\prime \prime}$.

The case of the Higgs boson decay in neutral currents has been briefly addressed for the signal alone in ref. [16] with the study of the reaction $e^{+} e^{-} \rightarrow e^{+} e^{-} \nu_{e} \bar{\nu}_{e} u \bar{u}$. The aim of the present paper is to complete and extend the analysis of ref. [16] to include general $q \bar{q}$ neutral currents contributions and the effect of the contributions from undetectable different-flavour neutrinos, in such a way as to provide realistic predictions for processes $e^{+} e^{-} \rightarrow l^{+} l^{-} \nu \bar{\nu} q \bar{q}$ at the parton level. In the following, $b$-quark tagging will be assumed, leaving aside $b \bar{b}$ final states, which lead to an interplay between Higgs and top physics and will be studied elsewhere. Consisting of only two jets, the processes considered in the present paper are free from QCD backgrounds.

The outline of the paper is as follows. In Section 2 the physical process is presented and the main technical issues of the calculation are explained. In Section 3 several numerical results are shown and discussed and the potentials of full $6 f$ calculations are stressed. Finally, Section 4 contains the conclusions.

\section{Physical process and computing technique}

The production of an intermediate mass Higgs boson is studied in the process $e^{+} e^{-} \rightarrow q \bar{q} l^{+} l^{-} \nu \bar{\nu}$, where a sum is made over the contributions of the $u, d, c$ and $s$ quarks, of the three neutrinos and of $l=e, \mu, \tau$. Particular attention will be devoted to the signature $q \bar{q} e^{+} e^{-} \nu \bar{\nu}$.

One of the interesting features of this process is the presence of both 
charged current and neutral current contributions [13], which is a situation never studied before, since all the six-fermion signatures analysed in the literature [13]-[15] involve only charged current contributions. Moreover this class of processes receives contribution from diagrams with up to three $t$ channel gauge bosons. This feature is of particular interest because of the large centre-of-mass (c.m.) energy, $\sqrt{s}$, at which the NLC will operate. These topologies enhance the cross section with growing $s$. The capability to provide predictions for processes with many electrons and electron-neutrinos in the final state is therefore crucial to discuss NLC physics. The present study demonstrates the possibility to deal successfully with the dynamics calculation and phase-space integration of this class of final states. Another important property is that the process is free from QCD backgrounds because only two jets are produced. As a drawback, the total cross section is smaller than in the $6 f$ processes with four or six jets.

However, the sums over quark, charged lepton and neutrino flavours, as well as the combined action of different mechanisms of production (see fig. 1), contribute to give a significant size to the cross section, so that, assuming an integrated luminosity of $500 \mathrm{fb}^{-1} / \mathrm{yr}$ and a Higgs mass of, say, $185 \mathrm{GeV}$, more than 1000 events can be expected at a c.m. energy of $360 \mathrm{GeV}$ and more than 2000 at $800 \mathrm{GeV}$ (see fig. 5). In particular, as will be seen in the numerical results, the presence of the $t$-channel contributions of vector boson fusion gives an enhancement of the cross sections at very high energies.

The diagrams containing a resonant Higgs boson coupled to gauge bosons for the $q \bar{q} e^{+} e^{-} \nu \bar{\nu}$ final state are shown in fig. 1. As can be seen, there are four terms of the Higgs-strahlung type and two of the fusion type. At relatively low energies, $\sqrt{s} \leq 500 \mathrm{GeV}$, the process of Higgs-strahlung dominates and, in particular, the charged current term is the most important one. As the energy is increased, the $t$-channel terms of vector boson fusion become more and more important, as they grow with increasing $s$, and they are dominant above $500 \mathrm{GeV}$. The diagrams for the processes with $\mu^{+} \mu^{-}$or $\tau^{+} \tau^{-}$instead of $e^{+} e^{-}$and/or $\bar{\nu}_{\mu, \tau} \nu_{\mu, \tau}$ instead of $\bar{\nu}_{e} \nu_{e}$ in the final state are a subset of those illustrated here.

The full set of diagrams containing a physical Higgs boson coupled to gauge bosons includes also those shown in fig. 2. These contributions are non-resonant, as the Higgs is exchanged in the $t$-channel, and their size can 

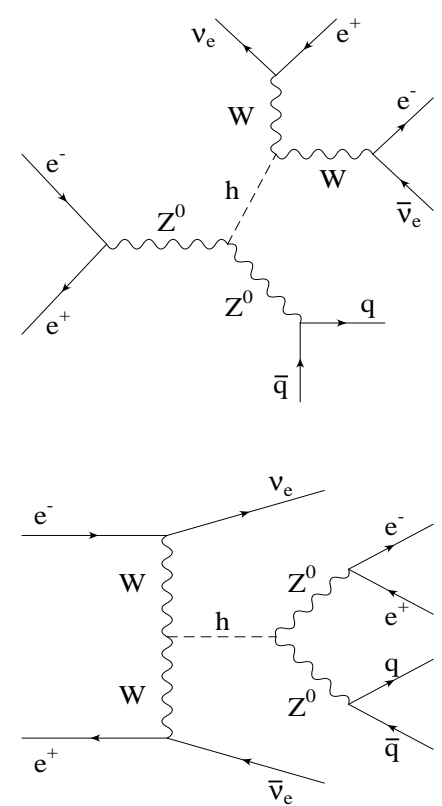
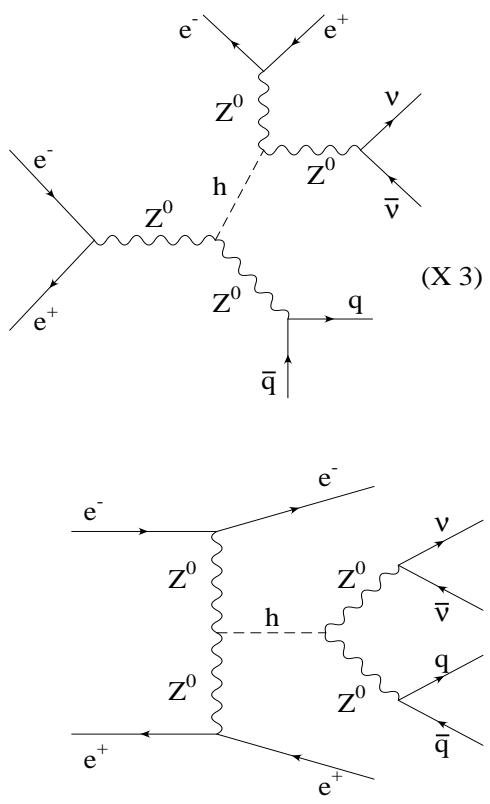

Figure 1: Feynman diagrams for the process $e^{+} e^{-} \rightarrow q \bar{q} e^{+} e^{-} \nu \bar{\nu}$ with a resonant Higgs boson.

be expected to be negligible at low energies; at high energies, however, they play an important rôle in preserving gauge invariance and unitarity of the $S$-matrix. This point will be discussed in more detail in the next section.

The total number of tree-level Feynman diagrams for the process under examination is of the order of one thousand, which makes the calculations very complicated for what concerns the determination of the transition matrix element as well as the phase-space integration.

The transition matrix element is calculated by means of ALPHA [20], an algorithm that allows the calculation of scattering amplitudes at the tree level without the use of Feynman diagrams. The results produced by this algorithm in a large number of calculations of multi-particle production are in full agreement with those obtained by programs using traditional techniques $[7,12,20]-[23]$. This fact may be considered as a significant test of ALPHA.

Anyway, also in the present work, checks have been made, reproducing by means of the helicity amplitude method [24] some of the results given by 

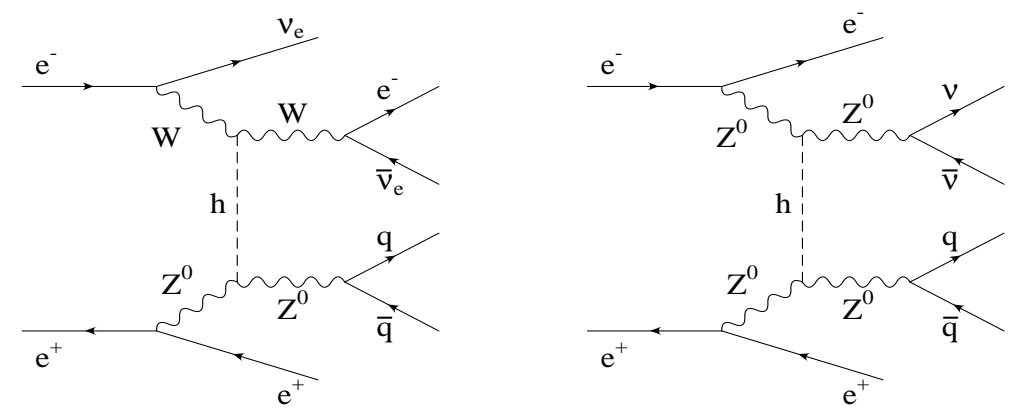

Figure 2: Feynman diagrams with a non-resonant Higgs boson (two similar diagrams are also obtained from these by particle exchanges).

ALPHA for the Higgs "signal" (which is defined below) and finding perfect agreement.

For the integration over the phase space, as was already done in refs. $[15,16]$, a code has been developed by adapting the Monte Carlo program HIGGSPV/WWGENPV [25, 26], originally developed to treat four-fermion production, to make $6 f$ calculations. The code can be used to perform Monte Carlo integrations and obtain cross sections, or to generate samples of unweighted events. Kinematical cuts can be introduced to simulate realistic experimental conditions. The effects of initial-state radiation (ISR) [27] and beamstrahlung [28] are taken into account by means of the standard convolution formula

$$
\sigma=\int d z_{1} d z_{2} D_{B S}\left(z_{1}, z_{2} ; s\right) \int d x_{1} d x_{2} D\left(x_{1}, s\right) D\left(x_{2}, s\right) \hat{\sigma}\left(z_{1}, z_{2} ; x_{1}, x_{2} ; s\right) .
$$

An accurate importance sampling procedure is required in the Monte Carlo integration to take care of the complicated structure of "singularities" in the integrand. This structure results from the presence of several mechanisms of Higgs production, and also from additional sources of variance among the very large number of background diagrams present in the matrix element.

The "singularities" given by different terms correspond to different regions of the (14-dimensional) phase space and in general must be treated with different sets of integration variables. As a consequence, a multichannel importance sampling technique is needed. If $n$ channels are introduced, the 

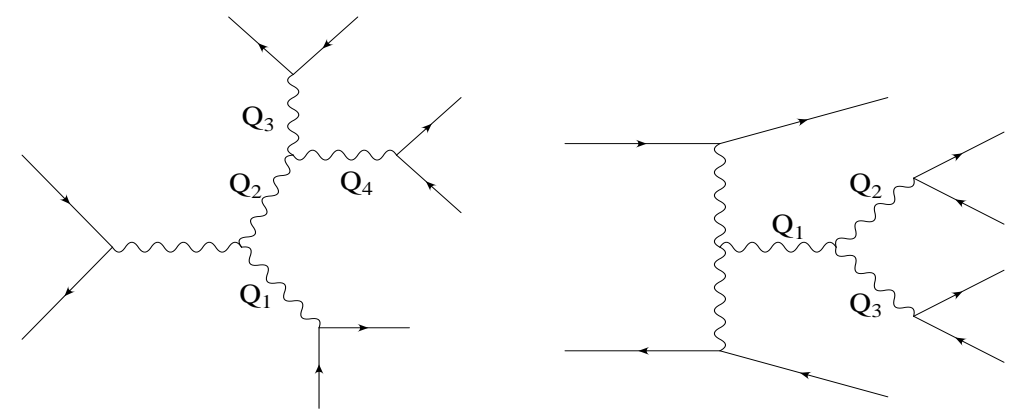

Figure 3: $s$-channel and $t$-channel topologies considered in the importance sampling.

integral is written as

$$
\int f(\mathbf{x}) d \mu(\mathbf{x})=\sum_{i=1}^{n} \int \frac{f\left(\mathbf{x}^{(i)}\right)}{p\left(\mathbf{x}^{(i)}\right)} p_{i}\left(\mathbf{x}^{(i)}\right) d \mu_{i}\left(\mathbf{x}^{(i)}\right), \quad p(\mathbf{x})=\sum_{i=1}^{n} p_{i}(\mathbf{x}),
$$

where each $\mathbf{x}^{(i)}$ is a set of integration variables with a corresponding measure $d \mu_{i}$, and $p_{i}$ is a suitably normalized distribution function for the importance sampling in the $i$-th channel.

The choice of integration variables is made within two kinds of phasespace decompositions, corresponding to two diagram topologies: $s$-channel, based on the Higgs-strahlung terms, and $t$-channel, based on the fusion terms, as illustrated in fig. 3 .

For the $s$-channel topology the phase-space decomposition reads:

$$
\begin{aligned}
d \Phi_{6}\left(P ; q_{1}, \ldots, q_{6}\right)= & (2 \pi)^{12} d \Phi_{2}\left(P ; Q_{1}, Q_{2}\right) d \Phi_{2}\left(Q_{1} ; q_{1}, q_{2}\right) \\
& d \Phi_{2}\left(Q_{2} ; Q_{3}, Q_{4}\right) d \Phi_{2}\left(Q_{3} ; q_{3}, q_{4}\right) d \Phi_{2}\left(Q_{4} ; q_{5}, q_{6}\right) \\
& d Q_{1}^{2} d Q_{2}^{2} d Q_{3}^{2} d Q_{4}^{2}
\end{aligned}
$$

where $P$ is the initial total momentum, $q_{i}$ are the momenta of the outgoing particles, while $Q_{i}$ are those of the internal particles. The notation $d \Phi_{2}\left(Q_{i} ; Q_{j}, Q_{k}\right)$ indicates the two-particle phase space; the momenta of the final particles $Q_{j}, Q_{k}$ are first generated in the rest frame of the particle of momentum $Q_{i}$ and then boosted to the laboratory frame. The integration variables are the four invariant masses $Q_{1}^{2}, \ldots, Q_{4}^{2}$ and five pairs of angular 
variables $\cos \theta_{i}, \phi_{i}$, one for each $d \Phi_{2}$ term. The invariant masses are sampled according to Breit-Wigner distributions of the form

$$
\frac{N}{\left(M^{2}-Q^{2}\right)^{2}+\Gamma^{2} M^{2}},
$$

given by the propagators of the Higgs or gauge bosons in the internal lines ( $N$ is a normalization factor). For the angular variables a flat distribution is assumed. The various $s$-channel terms differ for permutations of the external momenta and for the parameters $\Gamma, M$ in the importance sampling distributions.

In the case of $t$-channel diagrams, the phase space is

$$
\begin{aligned}
d \Phi_{6}\left(P ; q_{1}, \ldots, q_{6}\right)= & (2 \pi)^{9} d \Phi_{3}\left(P ; Q_{1}, q_{1}, q_{2}\right) \\
& d \Phi_{2}\left(Q_{1} ; Q_{2}, Q_{3}\right) d \Phi_{2}\left(Q_{2} ; q_{3}, q_{4}\right) d \Phi_{2}\left(Q_{3} ; q_{5}, q_{6}\right) \\
& d Q_{1}^{2} d Q_{2}^{2} d Q_{3}^{2},
\end{aligned}
$$

where, as before, the $q_{i}$ are the outgoing momenta, while the $Q_{i}$ are internal time-like momenta. The integration variables are the three invariant masses, $Q_{1}^{2}, Q_{2}^{2}, Q_{3}^{2}$, three pairs of angular variables $\cos \theta, \phi$ relative to the three $d \Phi_{2}$ terms, and, for the three-body phase space $d \Phi_{3}$, one energy, $q_{1}^{0}$, and four angular variables, $\cos \theta_{1}, \phi_{1}, \cos \theta_{2}, \phi_{2}$. The invariant masses are sampled, as in the $s$-channel case, according to Breit-Wigner distributions; one angular variable in $d \Phi_{3}$, say $\cos \theta_{1}$, is sampled by means of the distribution

$$
\frac{N}{\left(M_{V}^{2}+\sqrt{s} q_{1}^{0}\left(1-\cos \theta_{1}\right)\right)^{2}+\Gamma_{V}^{2} M_{V}^{2}},
$$

corresponding to the propagator of one space-like gauge boson $(V=W, Z)$ emitted by the initial electron or positron (typically one of the bosons participating in the fusion into Higgs); in some channels, corresponding to background diagrams, also another angular variable $\cos \theta$ relative to a two-body term $d \Phi_{2}$, is sampled in a similar way, in order to take into account the "singularity" associated with a boson propagator. All other variables have flat distributions.

\section{Numerical results and discussion}

The numerical results presented in this section are obtained with the same set of phenomenological parameters as adopted in ref. [15]. Namely, the input 
parameters are $G_{\mu}, M_{W}$ and $M_{Z}$, and other quantities, such as $\sin ^{2} \theta_{W}, \alpha$ and the widths of the $W$ and $Z$ bosons, are computed at tree level in terms of these constants. The Higgs width includes the fermionic contributions $h \rightarrow \mu \mu, \tau \tau, c c, b b$, with running masses for the quarks (to take into account QCD corrections [8]), the gluonic contribution $h \rightarrow g g$ [8], and the twovector boson channel, according to ref. [29]. The denominators of the bosonic propagators are of the form $p^{2}-M^{2}+i \Gamma M$, with fixed widths $\Gamma$. As already discussed in ref. [15], the aim of this choice is to minimize the possible sources of gauge violation in the computation [30].

Such gauge violations have been studied by the same methods as were used in ref. [15]. In particular, for what concerns $S U(2)$ gauge symmetry, comparisons have been made with results in the so-called "fudge scheme" [31]. A disagreement has been found at the level of few per cent for a c.m. energy of $360 \mathrm{GeV}$. The disagreement vanishes at higher energies. By careful inspection of the various contributions, it has been checked that the deviation at lower energies is due to the well-known fact that a given fudge factor, close to a resonance, mistreats the contributions that do not resonate in the same channel. Concerning $U(1)$ invariance, a test has been performed by using different forms of the photon propagator and finding perfect agreement, up to numerical precision, in the values of the squared matrix element.

The first group of results discussed in this section refers to cross-section calculations, performed by using the program as an integrator of weighted events. The signature considered in the first plots of total cross section is $q \bar{q} l^{+} l^{-} \nu \bar{\nu}$, where, in addition to the sums over quark and neutrinos flavours already mentioned, there is a sum over $l=e, \mu, \tau$. All other results are instead relative to the signature $q \bar{q} e^{+} e^{-} \nu \bar{\nu}$. Some samples of unweighted events, obtained by using the code as a generator, are then analysed in the remaining part of this section.

\subsection{Total cross sections}

In fig. 4 the total cross section (including the contribution of all the treelevel Feynman diagrams) is shown for three values of the Higgs mass in the intermediate range, 165, 185 and $255 \mathrm{GeV}$, at energies between 360 and 800 $\mathrm{GeV}$. To make a first analysis, the following kinematical cuts are adopted: the invariant mass of the quark-antiquark pair and that of the charged lepton pair are required to be greater than $70 \mathrm{GeV}$, the angles of the two charged 


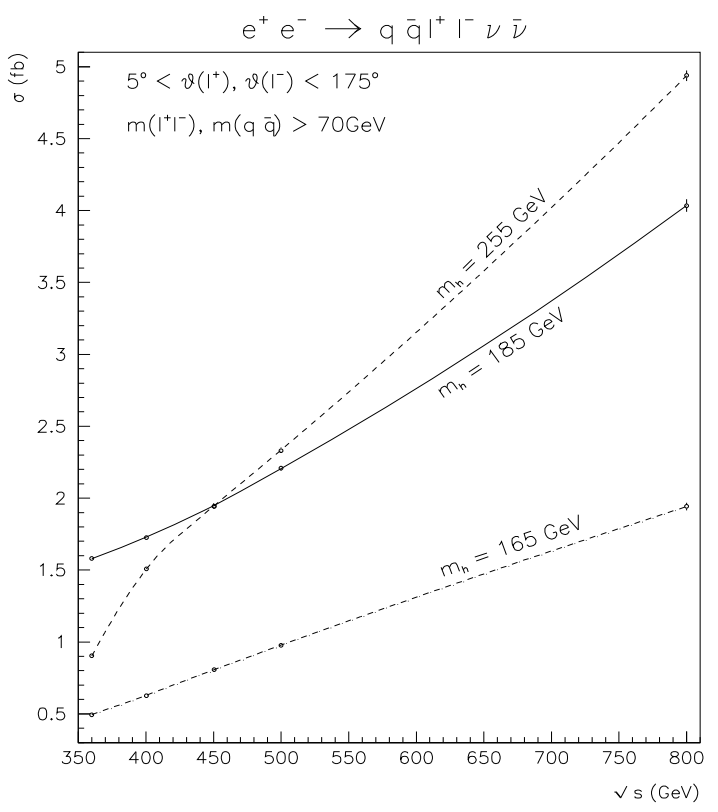

Figure 4: Total cross section for the process $e^{+} e^{-} \rightarrow q \bar{q} l^{+} l^{-} \nu \bar{\nu}$ in the Born approximation, as a function of $\sqrt{s}$ for three different values of the Higgs mass $m_{H}$. The angles $\theta\left(l^{+}\right), \theta\left(l^{-}\right)$of the charged leptons with the beam axis are in the interval $5^{\circ}-175^{\circ}$, the $e^{+} e^{-}$and the $q \bar{q}$ invariant masses are larger than $70 \mathrm{GeV}$.

leptons with respect to the beam axis within $5^{\circ}$ and $175^{\circ}$. This choice is applied to the quantities shown in figs. 4, 6 and 7 . Another set of cuts, with a lower limit of $20 \mathrm{GeV}$ on the $l^{+} l^{-}$invariant mass, is adopted in fig. 5 and in the study of event samples.

The increase with energy, common to all three curves in fig. 4, is due, at high energies, to the $t$-channel contributions; in the case of $m_{H}=255 \mathrm{GeV}$, the steep rise near $\sqrt{s}=360 \mathrm{GeV}$ is related to the existence of a threshold effect for the Higgs-strahlung process at an energy $\sqrt{s} \sim m_{H}+M_{Z}$.

In fig. 5 the total cross section is plotted, with the cut on the invariant mass of the charged lepton pair reduced to $20 \mathrm{GeV}$. The effect of this modification, as expected, is an enhancement of the cross section in the low-energy region: indeed the most important contribution at energies below $500 \mathrm{GeV}$ is given by the Higgs-strahlung diagram, with the Higgs decaying into two $W$ 


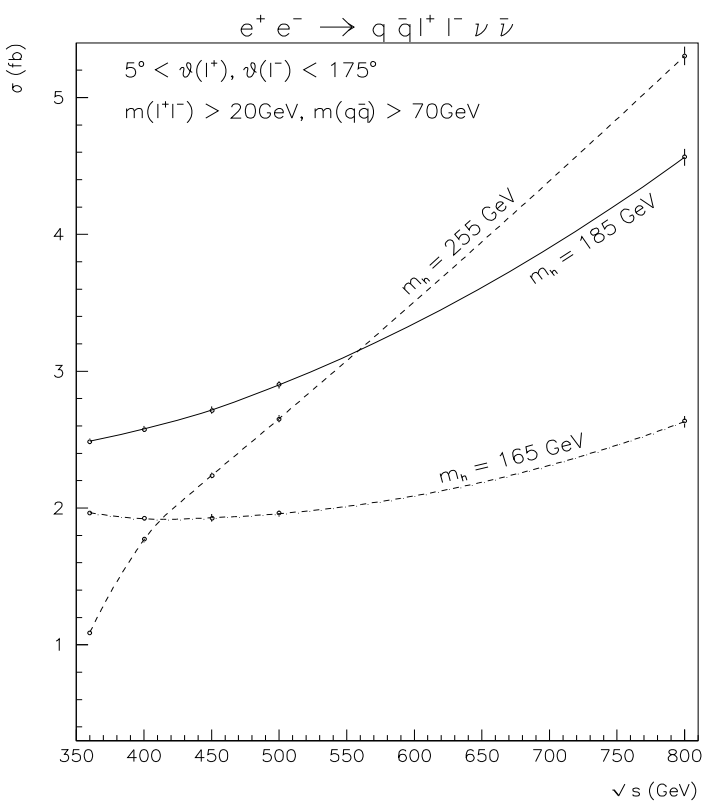

Figure 5: The same as in fig. 4 with the cut on the $l^{+} l^{-}$invariant mass reduced to $20 \mathrm{GeV}$.

bosons, which will be indicated from now on as the charged-current Higgsstrahlung diagram, and which is characterized by a broad distribution of the $l^{+} l^{-}$invariant mass that goes well below $70 \mathrm{GeV}$. This cut is still sufficient to reduce to a negligible size the contribution of virtual photon conversion into $l^{+} l^{-}$pairs. The behaviour of the cross section as the Higgs mass is varied depends on the interplay of the various production mechanisms and of the decay branchings involved; this behaviour can be better observed in the "signal" contribution that will be defined below (see fig. 9).

The effect of ISR is illustrated in fig. 6, for a Higgs mass of $185 \mathrm{GeV}$ and for the signature $q \bar{q} e^{+} e^{-} \nu \bar{\nu}$, to which all the remaining results refer. Here the $e^{+} e^{-}$invariant mass is again greater than $70 \mathrm{GeV}$.

The cross section in the presence of ISR is lowered by a quantity of the order of $10 \%$ with respect to the Born approximation. This phenomenon can be easily understood, since the initial-state radiation reduces the c.m. energy, so in this case it produces a shift towards energy values where the cross section is smaller. 


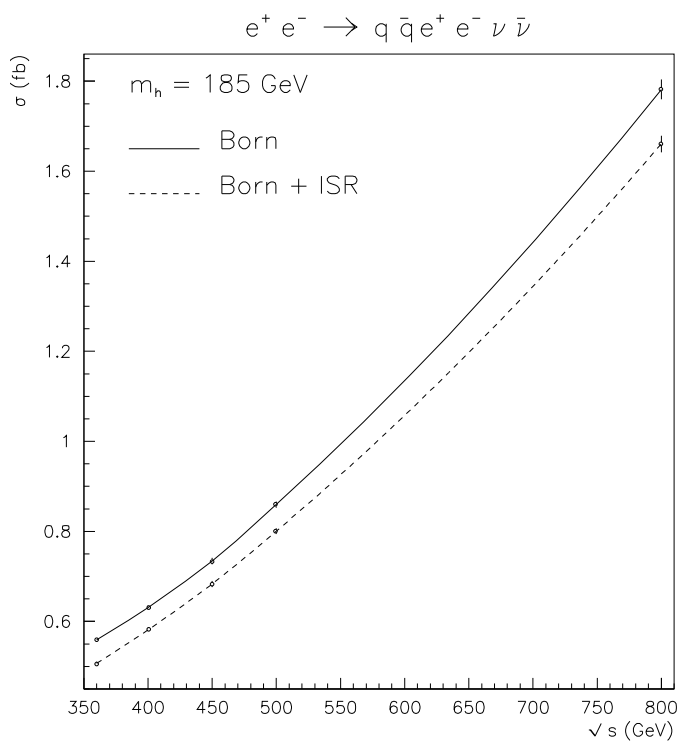

Figure 6: Effect of initial-state radiation on the total cross section of the process $e^{+} e^{-} \rightarrow q \bar{q} e^{+} e^{-} \nu \bar{\nu}$ as a function of $\sqrt{s}$ for a Higgs mass $m_{H}=185 \mathrm{GeV}$. Cuts are the same as in fig. 4.

\subsection{Definition and study of the Higgs signal}

The results discussed so far, as stated above, are given by the sum of all the tree-level Feynman diagrams. Strictly speaking, this is the only meaningful procedure. On the other hand, there is a number of reasons to consider a subset of diagrams that can be defined as the Higgs signal and to define a corresponding background. In the first place this is of great interest from the point of view of the search for the Higgs boson in the experiments. Moreover, as will be shown, such a definition allows one to make a comparison with results obtained in the narrow width approximation (NWA) [32]-[34], which are the only available estimations unless a complete $6 f$ calculation is performed. In principle, whenever a subset of diagrams is singled out, gauge invariance may be lost and unitarity problems may arise. However, in the following, an operative definition of signal and background is considered and its reliability is studied for various Higgs masses and c.m. energies.

The signal is defined as the sum of the six graphs containing a resonant Higgs boson, shown in fig. 1. The background is defined as the sum of all 
the diagrams without a Higgs boson. In this definition the diagrams with a non-resonant Higgs boson coupled to gauge bosons, shown in fig. 2, are missing both in the signal and in the background. Such a choice has been dictated by the fact that these non-resonant contributions cannot correctly be included in the signal, since they cannot find a counterpart in the NWA, and because of gauge cancellations with background contributions at high energies; however, as they depend on the Higgs mass, they should not be included in the background as well.

In order to give a quantitative estimate of the validity of this definition, the total cross section (sum of all the tree-level $6 f$ Feynman diagrams) is compared in fig. 7 with the incoherent sum of signal and background. The cuts are as in fig. 4 , in particular with the $e^{+} e^{-}$invariant mass greater than $70 \mathrm{GeV}$. In order to understand the meaning of these results, it is important to note that, as observed above, the contributions of the diagrams of fig. 2 are absent both from the signal and from the background: thus if we indicate these contributions to the scattering amplitude as $A_{h t}$, and the signal and background amplitudes as $A_{s}$ and $A_{b}$ respectively, the total squared amplitude is

$$
|A|^{2}=\left|A_{s}+A_{b}+A_{h t}\right|^{2}
$$

The terms neglected in the incoherent sum of signal and background are $\left|A_{h t}\right|^{2}$ and all the interference terms. Among these, the interferences of $A_{h t}$ with the rest are dominant at high energies as they involve gauge cancellations.

The curves of fig. 7 show that up to $500 \mathrm{GeV}$ the total cross section and the incoherent sum of signal and background are indistinguishable at a level of accuracy of $1 \%$, and the definition of signal may be considered meaningful; at higher energies, this separation of signal and background starts to be less reliable, since it requires to neglect effects that are relevant at this accuracy. In particular, at $800 \mathrm{GeV}$ the deviation is of the order of a few per cent and it decreases when the Higgs mass passes from 165 to 185 and to $255 \mathrm{GeV}$.

The above results are obtained with the set of kinematical cuts in which the $e^{+} e^{-}$invariant mass is greater than $70 \mathrm{GeV}$, but when this cut is at 20 $\mathrm{GeV}$, the difference between full cross section and incoherent sum of signal and background is significantly reduced (about $3-4 \%$ at $800 \mathrm{GeV}$ ). The analysis of event samples presented in the following is made within this latter set 

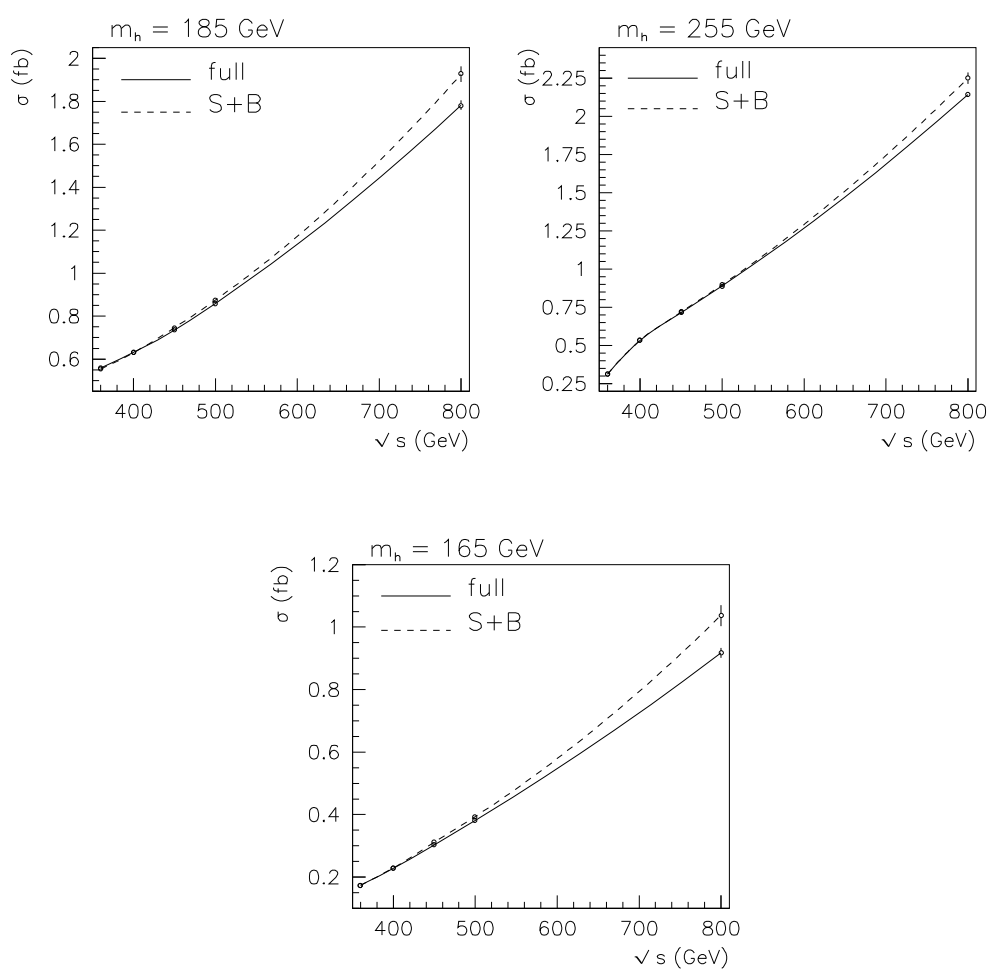

Figure 7: Full six-fermion cross section compared with the incoherent sum of "signal" (S) and "background" (B). A detailed discussion and an operative definition of "signal" and "background" are given in the text. 
of cuts, so that, up to $800 \mathrm{GeV}$, it can be considered reliable, at the level of accuracy of a few per cent, to speak of "background", as will be done.

On the other hand the problems arising when a definition of signal and background is attempted show the importance of a calculation involving the full set of tree-level Feynman diagrams to obtain reliable results, especially at high energies.

A comparison with the NWA is shown in fig. 8 for the processes $e^{+} e^{-} \rightarrow$ $q \bar{q} e^{+} e^{-} \nu \bar{\nu}$ and $e^{+} e^{-} \rightarrow q \bar{q} \mu^{+} \mu^{-} \nu \bar{\nu}$, where, for the sake of comparison, no kinematical cuts are applied and the results are in the Born approximation. Here $\sigma_{\text {sig }}$ is the signal cross section, containing the contributions of the six diagrams of fig. 1 (or the suitable subset of these for the case of the final state $\left.q \bar{q} \mu^{+} \mu^{-} \nu \bar{\nu}\right)$ and their interferences. The cross section in the NWA, $\sigma_{N W A}$, is obtained in the following way (for definiteness the case with $e^{+} e^{-}$in the final state is considered): the known cross sections for the processes of real Higgs production $e^{+} e^{-} \rightarrow h \nu \bar{\nu}, h e^{+} e^{-}[33,34]$ and $e^{+} e^{-} \rightarrow Z h$ [32] are multiplied by the appropriate branching ratios, so as to obtain six terms corresponding to the diagrams of fig. 1; then the incoherent sum of these terms is taken. Thus the comparison between $\sigma_{s i g}$ and $\sigma_{N W A}$ gives a measure of interference between the different production mechanisms and of off-shellness effects together.

As can be seen in fig. 8, the relative difference $R$ is of the order of some per cent, depending on the Higgs mass and the c.m. energy; in some cases it reaches values of more than $10 \%$, with no substantial difference between the two final states considered. In particular the off-shellness effects are much more important than the interference ones. In fact the relative size of the interferences has been separately evaluated by means of a comparison between $\sigma_{s i g}$ and the incoherent sum of the six diagrams of fig. 1 and has been found to be at most $2 \%$, but generally less than $1 \%$ for the c.m. energies and Higgs masses considered here.

The size of the off-shellness effects, comparable with the ISR lowering, indicates the importance of a full $6 f$ calculation in order to obtain sensible phenomenological predictions.

In fig. 9 the signal cross section is shown as a function of the Higgs mass for different c.m. energies. The behaviour is related to the branching ratios of the decays of the Higgs boson into gauge bosons and the differences between 


$$
R=2\left(\sigma_{\text {sig }}-\sigma_{\text {NWA }}\right) /\left(\sigma_{\text {sig }}+\sigma_{\text {NWA }}\right)
$$
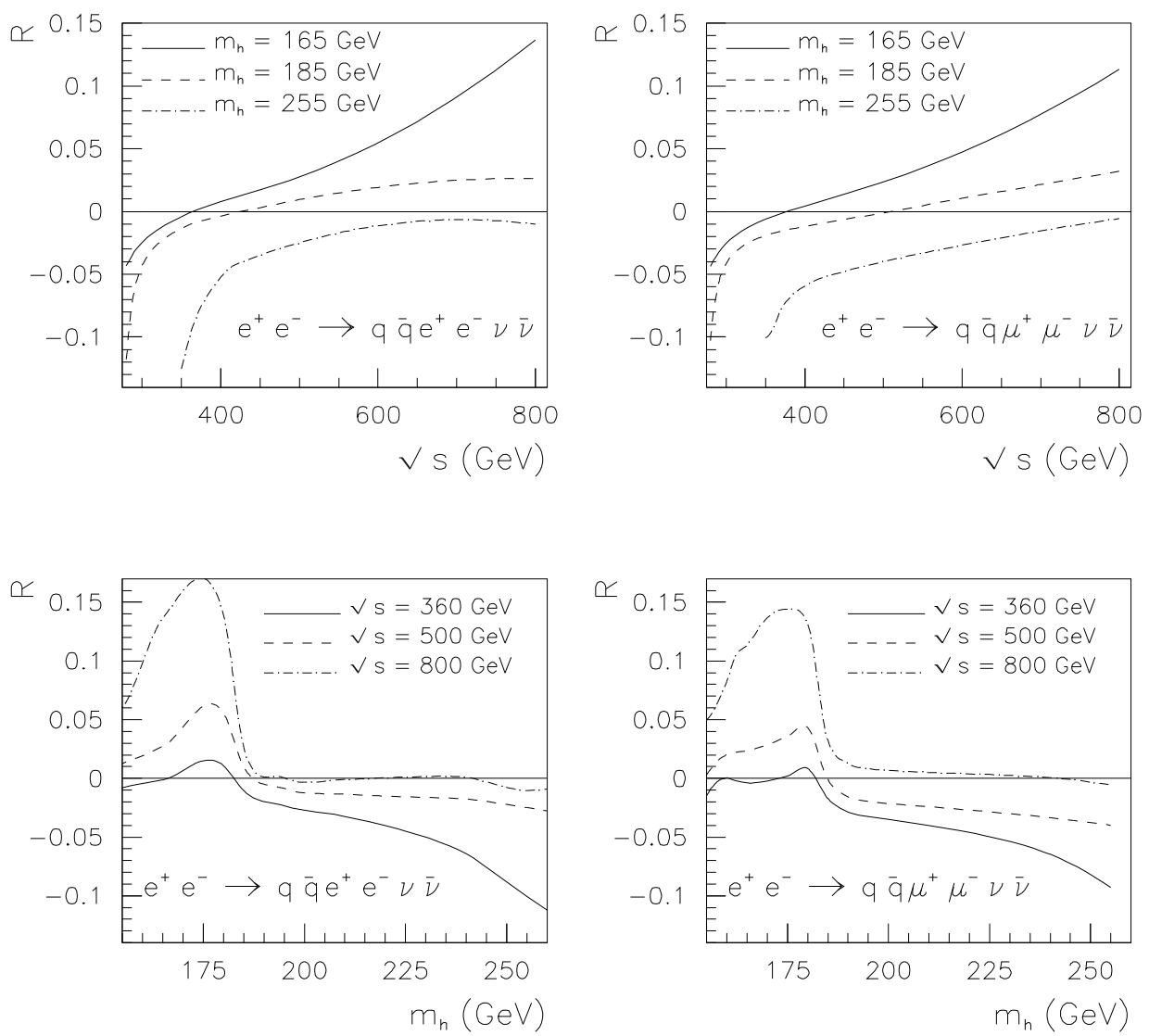

Figure 8: Comparison between the signal cross section obtained by a diagrammatic six-fermion calculation and the one calculated in the narrow width approximation (see the discussion in the text), as a function of $\sqrt{s}$ (upper row) and of the Higgs mass (lower row). 


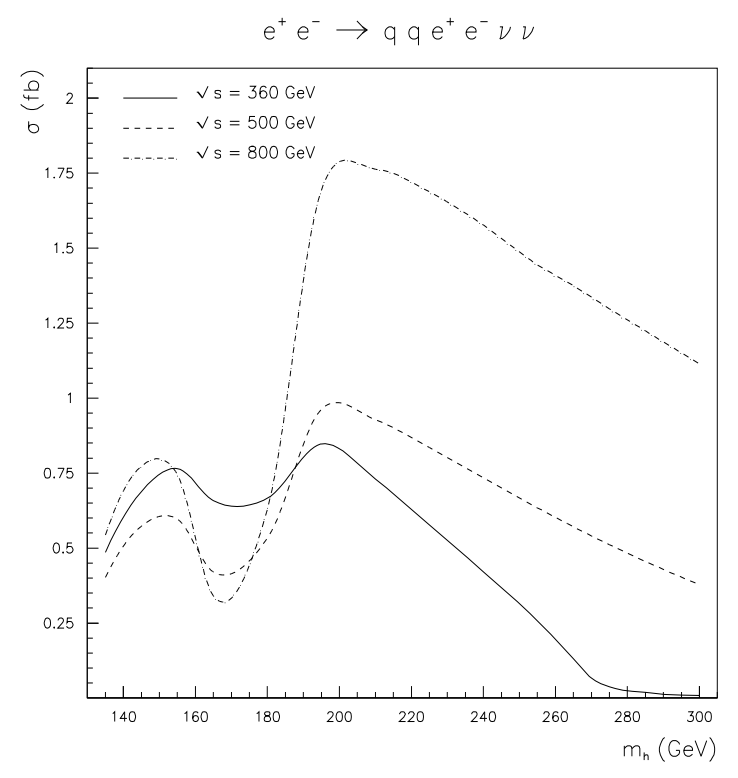

Figure 9: Signal cross section as a function of the Higgs mass for three different c.m. energies.

the three energy values considered are due to the variations in the relative sizes of the different signal contributions at different energies.

\subsection{Distributions}

The results presented in the following refer to samples of unweighted events for a Higgs mass of $185 \mathrm{GeV}$ at energies of 360 and $800 \mathrm{GeV}$, with or without the effect of ISR and beamstrahlung. The cuts adopted in all cases are the following: the invariant mass of the $q \bar{q}$ pair greater than $70 \mathrm{GeV}$, the invariant mass of the $e^{+} e^{-}$pair greater than $20 \mathrm{GeV}$, and the angles of the electron and positron with respect to the beam axis between $5^{\circ}$ and $175^{\circ}$; further cuts, applied in the analysis of particular cases, will be described later. The numbers of events in all the samples are normalized to the same luminosity.

In fig. 10 the invariant masses of two different systems of four momenta are studied at c.m. energies of 360 and $800 \mathrm{GeV}$ in the Born approximation (dashed histograms) as well as with ISR and beamstrahlung (solid his- 
tograms). The first set is given by $e^{+} e^{-}+$missing momentum, where the missing momentum is defined as $q_{\text {miss }}=p_{i n}^{+}+p_{\text {in }}^{-}-q_{e^{+}}-q_{e^{-}}-q_{q}-q_{\bar{q}}$. In the Born approximation this set of momenta corresponds to the system $e^{+} e^{-} \nu \bar{\nu}$. The other set considered is that corresponding to the four-fermion system $q \bar{q} e^{+} e^{-}$. As can be seen in fig. 1, these are two of the possible sets of four fermions produced by the decay of the Higgs boson in the process under consideration; there is also a third set, $q \bar{q} \nu \bar{\nu}$, whose invariant mass distribution, however, does not contain any new feature. The presence of the Higgs boson can be revealed by a peak in the distributions of these invariant masses. Indeed, in the Born approximation (dashed histograms), a sharp peak around $185 \mathrm{GeV}$ can be seen in each of the histograms of fig. 10. At a c.m. energy of $360 \mathrm{GeV}$, the most remarkable one is that of $e^{+} e^{-}+$missing momentum, associated to the system $e^{+} e^{-} \nu \bar{\nu}$, as it receives contributions from the charged current Higgs-strahlung diagram, which is dominant at this energy. In the presence of ISR and beamstrahlung, this peak is considerably lowered and broadened, while the other distribution, not involving the missing momentum, is not significantly affected by radiative effects. At $800 \mathrm{GeV}$ this phenomenon is even more evident, because the peak in the first distribution is completely eliminated by radiative effects, as a consequence of the small size of the charged current Higgs-strahlung term at this energy, while the second distribution results to be very sensitive to the presence of the Higgs, since it receives, around $185 \mathrm{GeV}$, contributions from the diagram of $W W$ fusion into Higgs, which is the dominant signal term at high energies, and the presence of ISR and beamstrahlung does not modify the shape of the histogram. Thus, at high energies, a very clean signal of the Higgs boson is provided by the process under study through this distribution.

The quantities analysed above are useful to reveal the presence of the Higgs boson and to determine its mass. Other variables can be considered to study the properties of this particle, such as spin and parity. Some examples are considered in figures 11,12 and 13.

When the process $e^{+} e^{-} \rightarrow H Z$ is considered, a variable that can give evidence of the scalar nature of the Higgs is the angle $\theta_{Z}$ of the $Z$ particle direction with respect to the beam in the laboratory frame. It is well known [32] that the differential cross section $d \sigma / d \cos \theta_{Z}$ goes as $\sin ^{2} \theta_{Z}$ at energies much greater than $M_{Z}$ and away from the threshold for Higgs production. A similar situation is expected to occur for the $6 f$ process under 

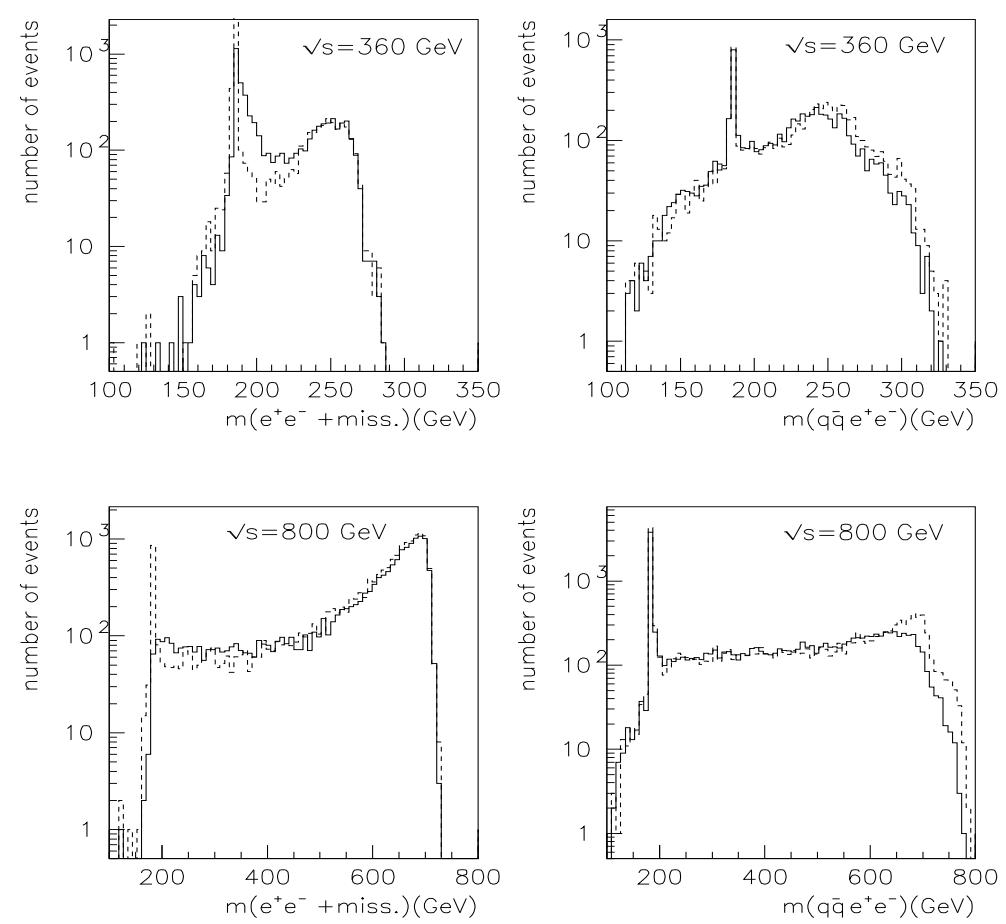

Figure 10: Invariant-mass distributions for four-fermion systems in the Born approximation (dashed histograms) and with ISR and beamstrahlung (solid histograms) at $\sqrt{s}=360 \mathrm{GeV}$ (upper row) and $\sqrt{s}=800 \mathrm{GeV}$ (lower row). 

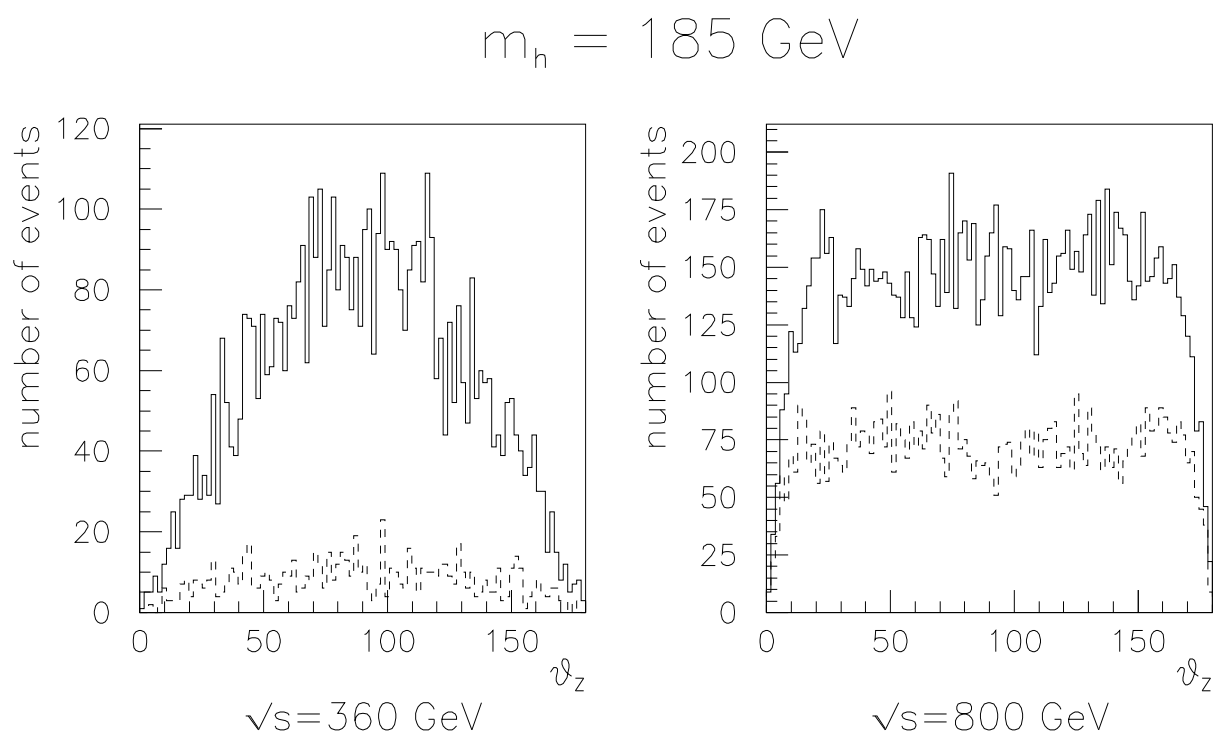

Figure 11: Distribution of the angle $\theta_{Z}$ of the $q \bar{q}$ pair with respect to the beam in the laboratory frame at $\sqrt{s}=360,800 \mathrm{GeV}$. The solid histogram represents the full calculation, the dashed histogram is the contribution of the background.

study when the Higgs-strahlung contributions are dominant. The distribution $d \sigma / d \theta_{Z}$ is shown, at the c.m. energies of 360 and $800 \mathrm{GeV}$, in fig. 11, where the $Z$ particle is reconstructed as the sum of the quark and antiquark momenta (indeed this is the case for the dominant diagram). The contribution from the background alone (dashed histogram) is also shown. The shape of the solid histogram shows the expected behaviour at $360 \mathrm{GeV}$, where Higgs-strahlung dominates.

At the c.m. energy of $800 \mathrm{GeV}$, where the dominant signal diagram is $W W$ fusion into Higgs, the situation is substantially different, since the process of Higgs production is of the $t$-channel type. One variable that results very sensitive to the presence of the Higgs boson is shown in fig. 12 and indicated as $\cos \theta_{Z Z} ; \theta_{Z Z}$ is the angle between the three-momenta in the laboratory frame of the $q \bar{q}$ and $e^{+} e^{-}$pairs, which correspond, in the diagram of $W W$ fusion, to the $Z$ particles coming from the Higgs. The full distribution 


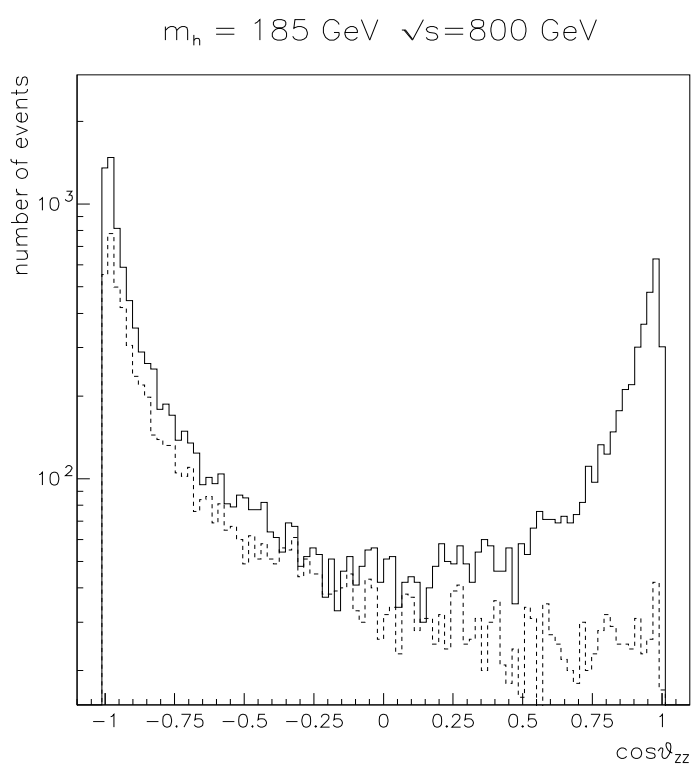

Figure 12: Distribution of the angle $\theta_{Z Z}$ between the $q \bar{q}$ and the $e^{+} e^{-}$pairs in the laboratory frame at $\sqrt{s}=800 \mathrm{GeV}$. The solid histogram is the full calculation, the dashed histogram is the background.

(solid line) and the contribution from the background alone (dashed line) are particularly distinguished in the region near 1 . There is here a clear signal of the presence of the Higgs, and such a variable can be used to impose kinematical cuts to single out signal contributions. This phenomenon is however of a kinematical nature, and is not directly related to the scalar nature of the Higgs, but is rather a consequence of the smallness of diagrams with the same topology of the $W W$ fusion in the background.

Another variable has been considered at the energy of $800 \mathrm{GeV}$ in fig. 13 . It is the angle $\theta_{Z}^{*}$ of the $Z$ particle, reconstructed as the sum of the electron and positron momenta, with respect to the beam, in the rest frame of the system $q \bar{q} e^{+} e^{-}$. The reference diagram is always the $W W$ fusion: this may be regarded asymptotically as an $s$-channel $W W$ scattering into $Z Z$, and, in the rest frame of the incoming $W W$ pair, the angular distribution of the produced $Z Z$ pair is determined by the scalar nature of the exchanged particle. In the first row of fig. 13 the plot on the left is made without additional cuts, 
while the plot on the right is obtained with the requirement $\cos \theta_{Z Z}>0$, so as to reduce the background. In the second row the invariant mass of $q \bar{q} e^{+} e^{-}$is required to be smaller than $250 \mathrm{GeV}$ (left) and within $20 \mathrm{GeV}$ around the Higgs mass (right) in order to further suppress the background. A clear difference between the shape of the full distribution and that of the background can in fact be seen, and in the last three plots the behaviour is very similar to the $\sin \theta_{Z}^{*}$ distribution expected on the basis of the above observations.

\section{Conclusions}

The processes $e^{+} e^{-} \rightarrow q \bar{q} l^{+} l^{-} \nu \bar{\nu}$ have been studied in connection with the search for an intermediate-mass Higgs boson. The study, which extends a previous analysis of $6 f$ signatures with only two jets, is characterized by the presence of neutral current contributions that were never considered before and by the fact that several mechanisms of Higgs production are simultaneously active.

The tool used for the numerical calculations is a Fortran code based on the algorithm ALPHA, for the determination of the scattering amplitude, and on a development of the Monte Carlo program HIGGSPV/WWGENPV, for the phase-space integration.

The total cross section, including all the tree-level Feynman diagrams, has been calculated with various kinematical cuts and taking into account the effects of ISR and beamstrahlung.

A definition of signal and background has been considered and its reliability has been studied. To this end the incoherent sum of "signal" and "background" has been compared with the full cross section, and this has shown deviations that, up to a c.m. energy of $500 \mathrm{GeV}$, are negligible to an accuracy of $1 \%$, but may be of several per cent at $800 \mathrm{GeV}$ (fig. 7). These deviations are, however, reduced when the kinematical selection criteria become more inclusive.

A comparison of the "signal" cross section with results in the NWA has shown that off-shellness effects have a relative size of several per cent (fig. 8).

The results of figs. 7 and 8 show the importance of a complete $6 f$ calculation to produce reliable results at the $\mathrm{TeV}$ scale. 


\section{$m_{h}=185 \mathrm{GeV} v \mathrm{~s}=800 \mathrm{GeV}$}
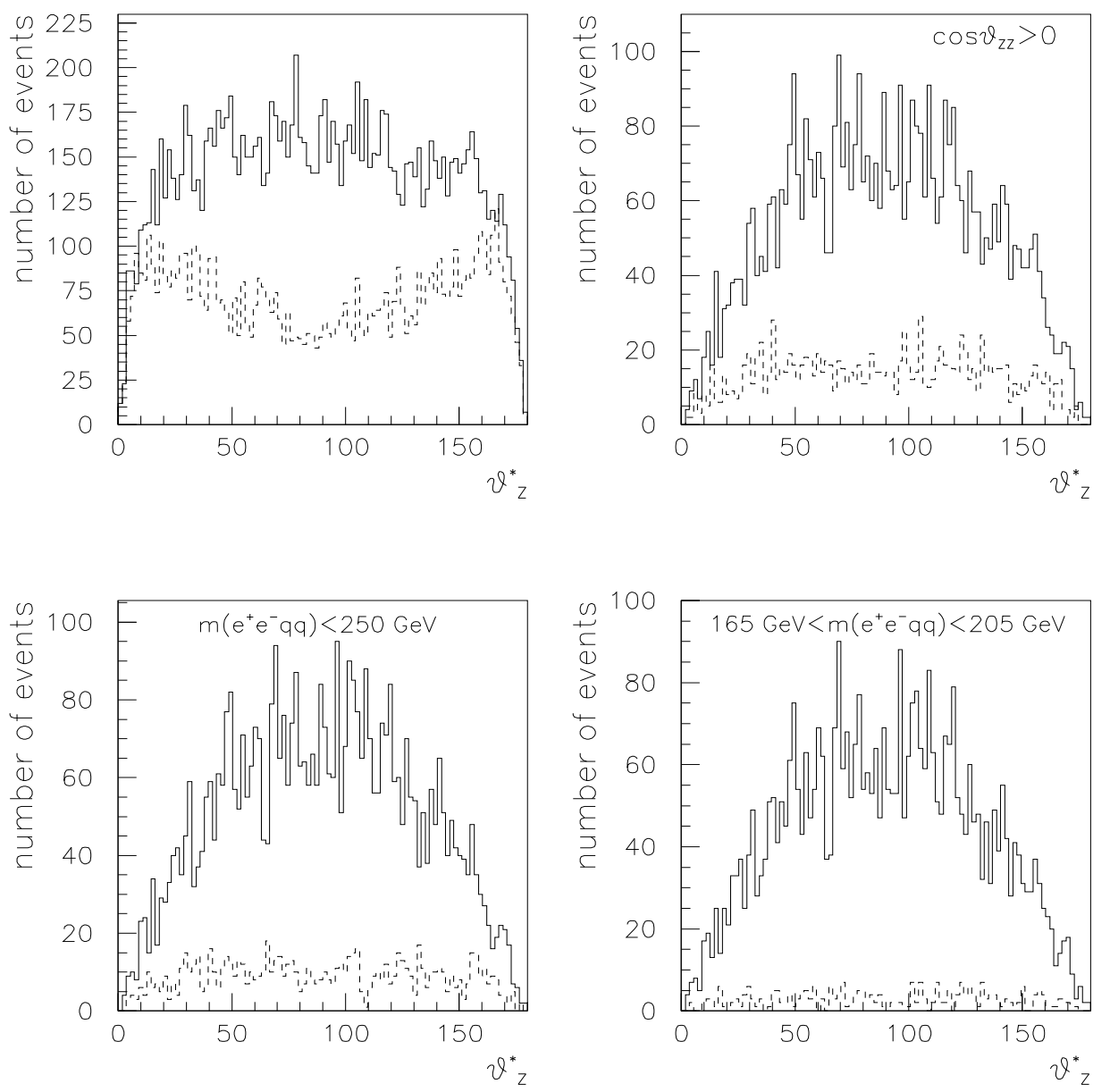

Figure 13: Distribution of the angle $\theta_{Z}^{*}$ of the $e^{+} e^{-}$pair with respect to the beam axis in the rest frame of the $q \bar{q} e^{+} e^{-}$system at $\sqrt{s}=800 \mathrm{GeV}$. The solid histogram is the full calculation, the dashed histogram is the background. First row: no additional cuts (left), $\cos \theta_{Z Z}>0$ (right), where the angle $\theta_{Z Z}$ is defined in the text and shown in fig. 12. Second row: $q \bar{q} e^{+} e^{-}$invariant mass smaller than $250 \mathrm{GeV}$ (left) and within $20 \mathrm{GeV}$ around the Higgs mass (right). 
In the study of generated events the problem of finding observables that are sensitive to the presence of the Higgs and to its properties has been addressed. The presence of several mechanisms of Higgs production, whose relative importance varies with energy, requires that different variables be considered according to the energy range studied.

The invariant masses of two sets of four fermions have been analysed first (fig. 10): one, relative to the system $e^{+} e^{-}+$missing momentum, is relevant to the detection of the Higgs boson at $360 \mathrm{GeV}$ of c.m. energy, but, at $800 \mathrm{GeV}$, the effects of ISR and beamstrahlung prevent to study the Higgs by means of this distribution. The other invariant mass, relative to the system $e^{+} e^{-} q \bar{q}$, is instead particularly useful at high energies and is almost completely unaffected by radiative effects.

Three angular variables have then been studied: the angle $\theta_{Z}$ (fig. 11) is suited to reveal the spin zero nature of the Higgs at $360 \mathrm{GeV}$, where the Higgs-strahlung dominates, but it gives no information at $800 \mathrm{GeV}$. The angles $\theta_{Z Z}$ (fig. 12) and $\theta_{Z}^{*}$ (fig. 13) are very useful at $800 \mathrm{GeV}$ : the first one is very effective to single out the signal, but is not able to distinguish the spin nature of the Higgs; the second one has a distribution whose shape is very different from that of the background, and is related to the spinless nature of the Higgs particle.

The computing strategy and the relative computer code developed in this work have been applied to study intermediate-mass Higgs physics. However, the variety of diagram topologies present in the matrix element and taken into account in the Monte Carlo integration, as well as the possibility provided by ALPHA of dealing with any kind of process, including now also QCD amplitudes, give the opportunity to examine other topics relevant to physics at future colliders, where $6 f$ production is involved.

\section{Acknowledgements}

We wish to thank A. Ballestrero and T. Ohl for discussions and for their interest in our work. The work of M. Moretti is funded by a Marie Curie fellowship (TMR-ERBFMBICT 971934). F. Gangemi thanks the INFN, Sezione di Pavia, for the use of computing facilities. 


\section{References}

[1] E. Accomando et al., Phys. Rep. 299 (1998) 1;

F. Boudjema, hep-ph/9809220.

[2] P. McNamara, talk given at the 29th International Conference on HighEnergy Physics (ICHEP 98), Vancouver, 1998.

[3] S. Mrenna and G.L. Kane, hep-ph/9406337;

S. Kim, S. Kuhlmann and W.M. Yao, Proceedings of 1996 DPF/DPB Summer Study on New Directions for High Energy Physics, Snowmass, 1996, p. 610; W.M. Yao, ibid., p. 619.

[4] Z. Kunszt, S. Moretti and W.J. Stirling, Z. Phys. C74 (1997) 479;

S. Moretti, hep-ph/9612310.

[5] D. Karlen, talk given at the 29th International Conference on HighEnergy Physics (ICHEP 98), Vancouver, 1998.

[6] G. Altarelli, T. Sjöstrand and F. Zwirner eds., Physics at LEP2, (CERN 96-01, Geneva, 1996), vols. 1 and 2.

[7] F. Boudjema and B. Mele (conveners), "Standard Model Processes" Working Group Report, in [6], vol. 1, p. 207.

[8] M. Carena and P.M. Zerwas (conveners), "Higgs Physics" Working Group Report, in [6], vol. 1, p. 351.

[9] M.L. Mangano and G. Ridolfi (conveners), "Event Generators for Discovery Physics" Working Group Report, in [6], vol. 2, p. 299.

[10] A. Ballestrero, Acta Phys. Pol. B29 (1998) 2811;

T. Ohl, talk given at the "Workshop on Physics and Detectors for Future $e^{+} e^{-}$Linear Colliders", Keystone, 1998.

[11] F. Berends and W. Beenakker (conveners), "WW Cross Sections and Distributions" Working Group Report, in [6] vol. 1, p. 79.

$[12]$ D. Bardin and R. Kleiss (conveners), "Event Generators for $W W$ Physics" Working Group Report, in [6], vol. 2, p. 3. 
[13] E. Accomando, A. Ballestrero and M. Pizzio, Nucl. Phys. B512 (1998) 19; in R. Settles ed., " $e^{+} e^{-}$Linear Colliders: Physics and Detector Studies, Part E", DESY 97-123E, p. 31, hep-ph/9807515.

[14] F. Yuasa, Y. Kurihara and S. Kawabata, Phys. Lett. B414 (1997) 178.

[15] G. Montagna, M. Moretti, O. Nicrosini and F. Piccinini, Eur. Phys. J. C2 (1998) 483.

[16] F. Gangemi, G. Montagna, M. Moretti, O. Nicrosini and F. Piccinini in R. Settles ed., " $e^{+} e^{-}$Linear Colliders: Physics and Detector Studies, Part E", DESY 97-123E, p. 393.

[17] S. Moretti, Phys. Lett. B420 (1998) 367, hep-ph/9808430.

[18] F. Caravaglios, M.L. Mangano, M. Moretti and R. Pittau, CERNTH/98-249, hep-ph/9807570, to appear in Nucl. Phys. B.

[19] P. Draggiotis, R.H.P. Kleiss and C.G. Papadopoulos, Phys. Lett. B439 (1998) 157.

[20] F. Caravaglios and M. Moretti, Phys. Lett. B358 (1995) 332.

[21] F. Caravaglios and M. Moretti, Z. Phys. C74 (1997) 291.

[22] M. Moretti, Nucl. Phys. B484 (1996) 3.

[23] G. Montagna, M. Moretti, O. Nicrosini and F. Piccinini, CERN-TH/98238, FNT/T-98/07, hep-ph/9807465, to appear in Nucl. Phys. B.

[24] P. De Causmaecker, R. Gastmans, W. Troost and T.T. Wu, Phys. Lett. B105 (1981) 215, Nucl. Phys. B206 (1982) 53;

F.A. Berends et al., Nucl. Phys. B206 (1982) 61, ibid. 239 (1984) 382, ibid. 239 (1984) 395, ibid. 264 (1986) 243, ibid. 264 (1986) 265;

M. Caffo and E. Remiddi, Helv. Phys. Acta 55 (1982) 339;

G. Passarino, Phys. Rev. D28 (1983) 2867, Nucl. Phys. B237 (1984) 249;

F.A. Berends, P.H. Daverveldt and R. Kleiss, Nucl. Phys. B253 (1985) 442 ;

R. Kleiss and W.J. Stirling Nucl. Phys. B262 (1985) 235, Phys. Lett. B179 (1986) 159. 
[25] Program HIGGSPV, by G. Montagna, O. Nicrosini and F. Piccinini, write up in $[9,12]$.

[26] Program WWGENPV, by G. Montagna, O. Nicrosini and F. Piccinini, write up in $[9,12]$. See also

G. Montagna, O. Nicrosini and F. Piccinini, Comput. Phys. Commun. 90 (1995) 141;

D.G. Charlton, G. Montagna, O. Nicrosini and F. Piccinini, Comput. Phys. Commun. 99 (1997) 355.

[27] E.A. Kuraev and V.S. Fadin, Sov. J. Nucl. Phys. 41 (1985) 466;

G. Altarelli and G. Martinelli, in Physics at LEP, J. Ellis and R. Peccei, eds., (CERN 86-02, Geneva, 1986), vol. 1, p. 47;

O. Nicrosini and L. Trentadue, Phys. Lett. B196 (1987) 551, Z. Phys. C39 (1988) 479;

F.A. Berends, G. Burgers and W.L. van Neerven, Nucl. Phys. B297 (1988) 429.

[28] T. Ohl, Comput. Phys. Commun. 101 (1997) 269.

[29] B. Kniehl, Phys. Lett. B244 (1990) 537.

[30] E.N. Argyres et al., Phys. Lett. B358 (1995) 339;

W. Beenakker et al., Nucl. Phys. B500 (1997) 255.

[31] U. Baur and D. Zeppenfeld, Phys. Rev. Lett. 75 (1995) 1002;

Y. Kurihara, D. Perret-Gallix and Y. Shimizu, Phys. Lett. B349 (1995) 367 ;

U. Baur, J.A.M. Vermaseren and D. Zeppenfeld, Nucl. Phys. B375 (1992) 3 .

[32] J. Ellis, M.K. Gaillard and D.V. Nanopoulos, Nucl. Phys. B106 (1976) 292 ;

J.D. Bjorken, SLAC Report 198 (1976).

[33] G. Altarelli, B. Mele and F. Pitolli, Nucl. Phys. B287 (1987) 205.

[34] M. Krämer, W. Kilian and P.M. Zerwas, Phys. Lett. B373 (1996) 135. 